\title{
Numerical renormalization group study of the symmetric Anderson-Holstein model: phonon and electron spectral functions
}

\author{
Gun Sang Jeon*, Tae-Ho Park, and Han-Yong Choi ${ }^{\dagger}$ \\ Department of Physics, BK21 Physics Research Division, \\ and Center for Nanotubes and Nanoscale Composites, \\ Sung Kyun Kwan University, Suwon 440-746, Korea.
}

\begin{abstract}
We study the symmetric Anderson-Holstein (AH) model at zero temperature with Wilson's numerical renormalization group (NRG) technique to study the interplay between the electronelectron and electron-phonon interactions. An improved method for calculating the phonon propagator using the NRG technique is presented, which turns out to be more accurate and reliable than the previous works in that it calculates the phonon renormalization explicitly and satisfies the boson sum rule better. The method is applied to calculate the renormalized phonon propagators along with the electron propagators as the onsite Coulomb repulsion $U$ and electron-phonon coupling constant $g$ are varied. As $g$ is increased, the phonon mode is successively renormalized, and for $g \gtrsim g_{c o}$ crosses over to the regime where the mode splits into two components, one of which approaches back to the bare frequency and the other develops into a soft mode. The initial renormalization of the phonon mode, as $g$ is increased from 0 , depends on $U$ and the hybridization $\Delta$; it gets softened (hardened) for $U \gtrsim(\lesssim) U_{s}(\Delta)$. Correlated with the emergence of the soft mode is the central peak of the electron spectral function severely suppressed. These NRG calculations will be compared with the standard Green's function results for the weak coupling regime to understand the phonon renormalization and soft mode.
\end{abstract}

\footnotetext{
* Present address: The Pennsylvania State University, Department of Physics, University Park, PA 16802.

$\dagger$ To whom the correspondences should be addressed. e-mail: hychoi@skku.ac.kr.
} 


\section{INTRODUCTION}

An important problem in interacting many particle systems is understanding the interplay between the electron-electron and electron-phonon interactions. Such interplay will be important to the problems such as the effects of phonons in the strongly correlated electron systems and the effects of electron correlations in the electron-phonon coupled systems. They include the cuprates, organic superconductors, Kondo systems, fullerenes, and the transition metal oxides, among others. Although some perturbative approaches have been proposed to understand the interplay [1] , there has not been much progress in this field partially because there are no reliable calculation schemes applicable in strong coupling regime where standard perturbation methods break down. Some non-perturbative techniques have been developed for strongly correlated impurity model in the context of the Kondo problem, but these techniques can not be applied to the more interesting correlated models on a lattice. With the recent advent of the dynamical mean-field theory (DMFT), however, those techniques can be and have been successfully applied to a wide class of lattice models. Within the DMFT, a lattice model is mapped onto an effective impurity model [2, 3]. Among the various methods applied to solve the effective impurity model, Wilson's numerical renormalization group (NRG) is particularly powerful in that it is non-perturbative in nature so that it can cover the whole parameter space, and it can be applied to both zero and finite temperatures and to both the dynamical and static properties [4, 5, 6, 7]. The NRG+DMFT technique has been successfully applied to the half-filled Hubbard model to study the metal-insulator transition at both $T=0$ and finite temperatures 8 , 9].

The prototype model for describing the interplay between electrons and phonons is the Hubbard-Holstein model and the Anderson-Holstein (AH) model which is the impurity version of the Hubbard-Holstein model. Recently, the AH model was studied at $T=0$ with the NRG technique by Hewson and Meyer [11]. The Holstein model was also studied with the DMFT combined with the NRG technique [12]. These works reported electron and phonon spectral functions among others. In the present work, we focus on the phonon propagator and report an improved method to calculate the phonon spectral function using the NRG technique for the AH model at zero temperature. This is the necessary first step for applying the DMFT to the Hubbard-Holstein model. In section II, we present the improved method for calculating the phonon spectral function, which turns out to be more accurate and reli- 
able than the previous methods in that it calculates the phonon renormalization explicitly and satisfies the sum rule better [11]. We will also present the standard Green's function analysis for the AH model, which provides a useful reference to understand the NRG results for the weak-coupling regime. Then, in section III, the results of these NRG calculations will be presented for the phonon and electron spectral functions, and they will be compared with the standard Green's function results to understand the phonon renormalization and soft mode. Finally, section IV is for the summary and concluding remarks.

\section{FORMULATION}

The AH model is defined by

$$
\begin{aligned}
\mathcal{H}_{A H} & =\sum_{k, \sigma} \epsilon_{k} c_{k \sigma}^{\dagger} c_{k \sigma}+\epsilon_{f} \sum_{\sigma} f_{\sigma}^{\dagger} f_{\sigma}+U f_{\uparrow}^{\dagger} f_{\uparrow} f_{\downarrow}^{\dagger} f_{\downarrow}+\omega_{0}\left(a^{\dagger} a+\frac{1}{2}\right) \\
& +\sum_{k, \sigma} V_{k}\left(f_{\sigma}^{\dagger} c_{k \sigma}+c_{k \sigma}^{\dagger} f_{\sigma}\right)+g\left(a^{\dagger}+a\right)\left(\sum_{\sigma} f_{\sigma}^{\dagger} f_{\sigma}-1\right),
\end{aligned}
$$

where $\sigma$ is the spin index, $\epsilon_{f}$ the impurity level energy, and $V_{k}$ is the hybridization matrix element of the impurity $f_{\sigma}$ electron with the conduction electrons of the host metal described by the operators $c_{k \sigma}$. The $f$ electron has the onsite Coulomb repulsion $U$, and is linearly coupled with the Einstein phonon of frequency $\omega_{0}$ with the coupling constant $g$ at the impurity site. The coupling is taken to be proportional to $\left(\sum_{\sigma} f_{\sigma}^{\dagger} f_{\sigma}-1\right)$, where the factor of -1 renders the problem particle-hole symmetric for the symmetric AH model, that is, the electron spectral function which will be discussed below is an even function of $\omega$. The AH model of Eq. (11) can be rewritten as

$$
\begin{aligned}
\mathcal{H}_{A H} & =\left(\epsilon_{f}+\frac{U}{2}\right) \sum_{\sigma} f_{\sigma}^{\dagger} f_{\sigma}+\frac{U}{2}\left(\sum_{\sigma} f_{\sigma}^{\dagger} f_{\sigma}-1\right)^{2}+\omega_{0} a^{\dagger} a \\
& +\sum_{k, \sigma} \epsilon_{k} c_{k \sigma}^{\dagger} c_{k \sigma}+\sum_{k, \sigma} V_{k}\left(f_{\sigma}^{\dagger} c_{k \sigma}+c_{k \sigma}^{\dagger} f_{\sigma}\right)+g\left(a^{\dagger}+a\right)\left(\sum_{\sigma} f_{\sigma}^{\dagger} f_{\sigma}-1\right),
\end{aligned}
$$

disregarding constant terms. We will focus on the symmetric case of $\epsilon_{f}+U / 2=0$ in the present work for simplicity. Asymmetric cases are also straightforward.

The electron and phonon Green's functions on the impurity site are defined, respectively, by

$$
G_{\sigma}(\omega)=\left\langle\left\langle f_{\sigma}, f_{\sigma}^{\dagger}\right\rangle\right\rangle_{\omega}, \quad d(\omega)=\left\langle\left\langle a, a^{\dagger}\right\rangle\right\rangle_{\omega},
$$


where the correlator $\langle\langle\rangle$ is defined as

$$
\begin{aligned}
\left\langle\left\langle\mathcal{O}_{1}, \mathcal{O}_{2}\right\rangle\right\rangle_{\omega} & =\int_{-\infty}^{\infty} d t e^{i \omega t}\left\langle\left\langle\mathcal{O}_{1}, \mathcal{O}_{2}\right\rangle\right\rangle_{t} \\
\left\langle\left\langle\mathcal{O}_{1}, \mathcal{O}_{2}\right\rangle\right\rangle_{t} & =-i \theta(t)\left\langle\left[\mathcal{O}_{1}(t), \mathcal{O}_{2}(0)\right]_{\zeta}\right\rangle=-i \theta(t)\left\langle\left[\mathcal{O}_{1}(0), \mathcal{O}_{2}(-t)\right]_{\zeta}\right\rangle .
\end{aligned}
$$

Here, $\theta$ is the step function, \langle\rangle the thermodynamic average, and $\zeta=+$ if both $\mathcal{O}_{1}$ and $\mathcal{O}_{2}$ are fermion operators and $\zeta=-$ otherwise. The equation of motion (EOM) of the correlator is given by

$$
\begin{aligned}
\omega\left\langle\left\langle\mathcal{O}_{1}, \mathcal{O}_{2}\right\rangle_{\omega}\right. & =\left\langle\left[\mathcal{O}_{1}, \mathcal{O}_{2}\right]_{\zeta}\right\rangle+\left\langle\left\langle\left[\mathcal{O}_{1}, \mathcal{H}\right]_{-}, \mathcal{O}_{2}\right\rangle_{\omega}\right. \\
& =\left\langle\left[\mathcal{O}_{1}, \mathcal{O}_{2}\right]_{\zeta}\right\rangle-\left\langle\left\langle\mathcal{O}_{1},\left[\mathcal{O}_{2}, \mathcal{H}\right]_{-}\right\rangle_{\omega}\right.
\end{aligned}
$$

The $f$-electron Green's function can be written using the EOM [11, 13]

$$
G_{\sigma}^{-1}(\omega)=\omega-\epsilon_{f}-\bar{\Delta}(\omega)-\Sigma_{\sigma}^{U}(\omega)-\Sigma_{\sigma}^{g}(\omega)
$$

where the self-energy corrections are given by

$$
\bar{\Delta}(\omega)=\sum_{k} \frac{V_{k}^{2}}{\omega-\epsilon_{k}}, \quad \Sigma_{\sigma}^{U}(\omega)=U \frac{\left\langle\left\langle f_{\sigma} f_{-\sigma}^{\dagger} f_{-\sigma}, f_{\sigma}^{\dagger}\right\rangle\right\rangle_{\omega}}{\left\langle\left\langle f_{\sigma}, f_{\sigma}^{\dagger}\right\rangle\right\rangle_{\omega}}, \quad \Sigma_{\sigma}^{g}(\omega)=g \frac{\left\langle\left\langle f_{\sigma}\left(a^{\dagger}+a\right), f_{\sigma}^{\dagger}\right\rangle_{\omega}\right.}{\left\langle\left\langle f_{\sigma}, f_{\sigma}^{\dagger}\right\rangle_{\omega}\right.} .
$$

This form of defining $G_{\sigma}(\omega)$ was proposed and its advantages over the direct NRG calculation of $\left\langle\left\langle f, f^{\dagger}\right\rangle\right\rangle_{\omega}$ were discussed by Bulla et al. and Hewson and Meyer [11, 13].

Now, we consider the phonon propagator $d(\omega)$ defined by Eq. (3). There are several ways to calculate the phonon propagator: (a) We may calculate $d(\omega)=\left\langle\left\langle a, a^{\dagger}\right\rangle\right\rangle_{\omega}$ directly by NRG, or, (b) calculate

$$
d^{-1}(\omega)=d_{0}^{-1}(\omega)-g \frac{\left\langle\left\langle n_{f}, a^{\dagger}\right\rangle\right\rangle_{\omega}}{\left\langle\left\langle a, a^{\dagger}\right\rangle\right\rangle_{\omega}}
$$

where

$$
d_{0}^{-1}(\omega)=\omega-\omega_{0}, \quad n_{f}=\sum_{\sigma} f_{\sigma}^{\dagger} f_{\sigma}-1
$$

or, (c) calculate

$$
d(\omega)=d_{0}(\omega)+\left[g d_{0}(\omega)\right]^{2}\left\langle\left\langle n_{f}, n_{f}\right\rangle\right\rangle_{\omega} .
$$

Eqs. (8) and (10) may be derived using the EOM as follows. From the Eq. (5), we have

$$
\left(\omega-\omega_{0}\right)\left\langle\left\langle a, a^{\dagger}\right\rangle_{\omega}=1+g\left\langle\left\langle n_{f}, a^{\dagger}\right\rangle_{\omega} .\right.\right.
$$


Eq. (11) can be rearranged, by dividing both sides by $\left\langle\left\langle a, a^{\dagger}\right\rangle\right\rangle_{\omega}$, to Eq. (8). Again from the EOM, we also obtain

$$
\omega\left\langle\left\langle n_{f}, a^{\dagger}\right\rangle\right\rangle_{\omega}=\omega_{0}\left\langle\left\langle n_{f}, a^{\dagger}\right\rangle\right\rangle_{\omega}+g\left\langle\left\langle n_{f}, n_{f}\right\rangle\right\rangle_{\omega}
$$

Substituting this into Eq. (11) yields Eq. (10).

The advantage of using Eq. (8) is that it is written in the form of Dyson equation and shows the renormalization explicitly. The form of Eq. (10) was employed previously [11, 12]. It will be extremely difficult, however, to obtain the renormalized spectrum near $\omega \approx \omega_{0}$ from Eq. (10) because of the strong singularity of the $\left[d_{0}(\omega)\right]^{2}$. We therefore use Eq. (8) in the present analysis. This argument is made more quantitative by the sum rule. The important point is that the $d(\omega)$ must satisfy the boson sum rule:

$$
-\frac{1}{\pi} \int_{-\infty}^{\infty} d \omega \operatorname{Im} d(\omega)=1
$$

The three ways of calculating the phonon propagator above will be discussed in the following section. It will be shown that the method (b) satisfies the sum rule of Eq. (13) much better than the other methods.

Alternatively, one may use the phonon Green's function defined by

$$
D(\omega)=\left\langle\left\langle a+a^{\dagger}, a+a^{\dagger}\right\rangle\right\rangle_{\omega}
$$

We then have

$$
\begin{aligned}
D^{-1}(\omega) & =D_{0}^{-1}(\omega)-g \frac{\left\langle\left\langle n_{f}, a+a^{\dagger}\right\rangle\right\rangle_{\omega}}{\left\langle\left\langle a+a^{\dagger}, a+a^{\dagger}\right\rangle\right\rangle_{\omega}} \\
D(\omega) & =D_{0}(\omega)+\left[g D_{0}(\omega)\right]^{2}\left\langle\left\langle n_{f}, n_{f}\right\rangle\right\rangle_{\omega}
\end{aligned}
$$

which correspond to Eqs. (8) and ([10), respectively, where $D_{0}(\omega)=2 \omega_{0} /\left(\omega^{2}-\omega_{0}^{2}\right)$. There are essentially no differences between the phonon Green's functions $d(\omega)$ and $D(\omega)$. The advantage of $d(\omega)$ over $D(\omega)$ is that there exists the useful sum rule of Eq. (13) the $d(\omega)$ satisfies, while the sum rule the $D(\omega)$ satisfies

$$
-\frac{1}{\pi} \int_{-\infty}^{\infty} d \omega \operatorname{ImD}(\omega) n(\omega)=\left\langle\left(a^{\dagger}+a\right)\left(a^{\dagger}+a\right)\right\rangle
$$

where $n(\omega)$ is the Bose function, is less useful.

Before we present the detailed NRG results for the AH model, we consider the standard Green's function analyses of the model for the phonon and electron propagators. It will 
a)

a)

b)

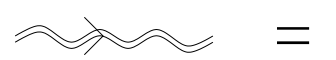

c)

$\alpha$

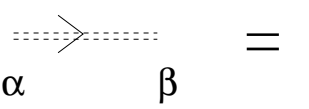

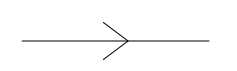
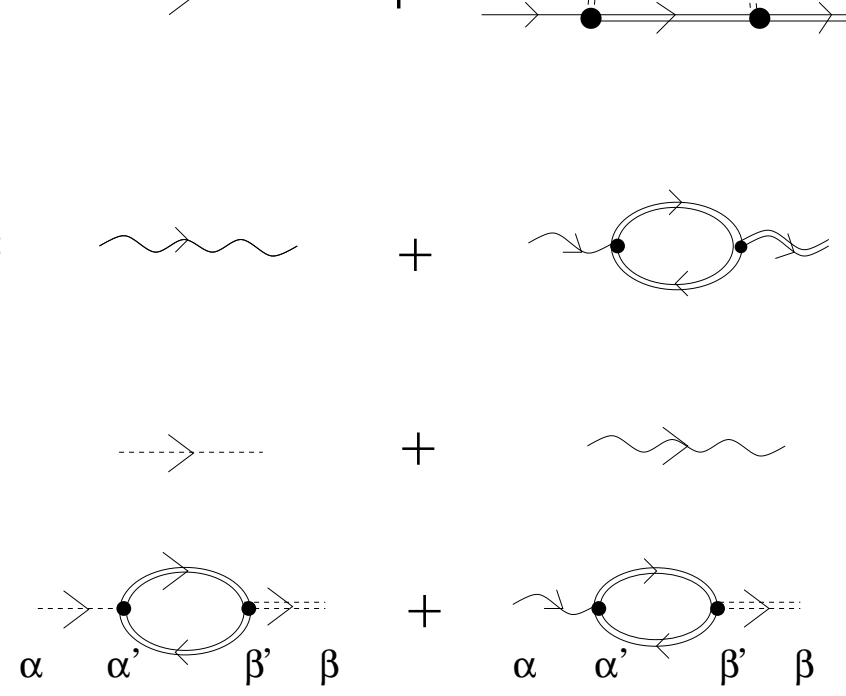

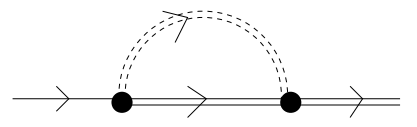

FIG. 1: The Feynman diagram for the Anderson-Holstein model. The single (double) solid and wiggle lines represent, respectively, the bare (renormalized) electron and phonon propagators. The single dashed line refers to the Hubbard interaction, and the double dashed line to the effective interaction between two electrons of spin $\alpha$ and $\beta$.

provide a useful reference to understand the NRG results for the weak-coupling regime. Within the perturbative Green's function scheme, the renormalized electron and phonon propagators for $U=0$ are, as shown in Fig. 1(a) and (b), given by

$$
G^{-1}(\omega)=G_{0}^{-1}(\omega)-\Sigma(\omega), \quad D^{-1}(\omega)=D_{0}^{-1}(\omega)-g^{2} \chi(\omega)
$$

where $G_{0}$ is the bare electron Green's function given by

$$
G_{0}(\omega)=\frac{1}{\omega+i \Delta}, \quad \Delta=\sum_{k} \pi\left|V_{k}\right|^{2} \delta\left(\omega-\epsilon_{k}\right)
$$

and the self-energy $\Sigma$ and the polarization $\chi$ are given by

$$
\begin{aligned}
& \Sigma(\omega)=\frac{1}{\pi^{2}} \int_{-\infty}^{\infty} d \epsilon \int_{-\infty}^{\infty} d \Omega \frac{f(\epsilon)+n(-\Omega)}{\epsilon+\Omega-\omega-i \delta} \operatorname{Im} G(\epsilon) \operatorname{Im} V(\Omega), \\
& \chi(\omega)=\frac{1}{\pi^{2}} \int_{-\infty}^{\infty} d \epsilon \int_{-\infty}^{\infty} d \epsilon^{\prime} \frac{f(\epsilon)-f\left(\epsilon^{\prime}\right)}{\omega+\epsilon-\epsilon^{\prime}+i \delta} \operatorname{Im} G(\epsilon) \operatorname{Im} G\left(\epsilon^{\prime}\right) .
\end{aligned}
$$

$\Delta$ of Eq. (18) is the minus imaginary part of $\bar{\Delta}$ defined in Eq. (而), $\bar{\Delta}=-i \Delta . V$ is the effective interaction between two electrons which is reduced to $V=g^{2} D$ for $U=0$ as discussed below. We consider the case where $\Delta(\omega)$ is a constant. At $T=0$ and up to the 
2nd order in $g$, we obtain

$$
\begin{aligned}
\chi(\omega) & =-\frac{1}{\pi} \int_{0}^{\infty} d \epsilon \frac{\Delta}{\epsilon^{2}+\Delta^{2}}\left(\frac{1}{\epsilon+\omega+i \Delta}+\frac{1}{\epsilon-\omega-i \Delta}\right) \\
& =\frac{2 \Delta}{\pi \omega} \frac{1}{\omega+i 2 \Delta} \ln \left(1-i \frac{\omega}{\Delta}\right) .
\end{aligned}
$$

The real and imaginary parts are given, respectively, by

$$
\begin{aligned}
& \operatorname{Re\chi }(\omega)=\frac{\Delta}{\pi} \frac{1}{4 \Delta^{2}+\omega^{2}}\left[\ln \left(1+\frac{\omega^{2}}{\Delta^{2}}\right)-\frac{4 \Delta}{\omega} \tan ^{-1} \frac{\omega}{\Delta}\right], \\
& \operatorname{Im\chi }(\omega)=-\frac{2 \Delta^{2}}{\pi \omega} \frac{1}{4 \Delta^{2}+\omega^{2}}\left[\ln \left(1+\frac{\omega^{2}}{\Delta^{2}}\right)+\frac{\omega}{\Delta} \tan ^{-1} \frac{\omega}{\Delta}\right] .
\end{aligned}
$$

For $\omega \ll \Delta$, it is reduced to

$$
\chi(\omega) \approx-\frac{1}{\pi \Delta}\left(1-\frac{5}{6} \frac{\omega^{2}}{\Delta^{2}}+i \frac{\omega}{\Delta}\right),
$$

which will be used in discussing the soft phonon mode in the following section.

When $U \neq 0$, the renormalized phonon propagator is given by

$$
D^{-1}(\omega)=D_{0}^{-1}(\omega)-g^{2} \frac{\chi(\omega)}{1-U \chi(\omega) / 2} .
$$

This may be obtained as follows. The effective interaction between the $f$-electrons $V_{\alpha \beta}$, where $\alpha$ and $\beta$ are the spin indices, due to both electron-phonon and electron-electron interactions is determined, as shown in Fig. 1(c), by

$$
V_{\alpha \beta}(i \omega)=V_{\alpha \beta}^{0}+\sum_{\alpha^{\prime}, \beta^{\prime}} V_{\alpha \alpha^{\prime}}^{0} \chi_{\alpha^{\prime} \beta^{\prime}}(i \omega) V_{\beta^{\prime} \beta}(i \omega)
$$

where

$$
V_{\alpha \alpha}^{0} \equiv V^{0}=g^{2} D_{0}, \quad V_{\alpha,-\alpha}^{0}=U+V^{0}
$$

The solution to Eq. (24) is given by

$$
\begin{aligned}
& V_{\uparrow \uparrow}=V_{\downarrow \downarrow}=\frac{V^{0}+U\left(2 V^{0}+U\right) \chi_{\uparrow \uparrow}}{\left[1-\left(2 V^{0}+U\right)\left(\chi_{\uparrow \uparrow}+\chi_{\uparrow \downarrow}\right)\right]\left[1+U\left(\chi_{\uparrow \uparrow}-\chi_{\uparrow \downarrow}\right)\right]}, \\
& V_{\uparrow \downarrow}=V_{\downarrow \uparrow}=\frac{V^{0}+U-U\left(2 V^{0}+U\right) \chi_{\uparrow \downarrow}}{\left[1-\left(2 V^{0}+U\right)\left(\chi_{\uparrow \uparrow}+\chi_{\uparrow \downarrow}\right)\right]\left[1+U\left(\chi_{\uparrow \uparrow}-\chi_{\uparrow \downarrow}\right)\right]} .
\end{aligned}
$$

We put $\chi_{\uparrow \uparrow}=\chi_{\downarrow \downarrow}=\chi / 2$ and $\chi_{\uparrow \downarrow}=\chi_{\downarrow \uparrow}=0$ to obtain

$$
\begin{aligned}
& V_{\uparrow \uparrow}=\frac{V^{0}+U\left(V^{0}+U / 2\right) \chi}{\left[1-\left(V^{0}+U / 2\right) \chi\right][1+U \chi / 2]}, \\
& V_{\uparrow \downarrow}=\frac{V^{0}+U}{\left[1-\left(V^{0}+U / 2\right) \chi\right][1+U \chi / 2]} .
\end{aligned}
$$


The renormalized electron-phonon interaction is then given by [10]

$$
\begin{aligned}
V_{e l-p h} & =V_{\uparrow \uparrow}-\frac{U^{2} \chi / 2}{1-U^{2} \chi^{2} / 4}=V_{\uparrow \downarrow}-\frac{U}{1-U^{2} \chi^{2} / 4} \\
& =\frac{V^{0}}{\left[1-\left(2 V^{0}+U\right) \chi / 2\right][1-U \chi / 2]} .
\end{aligned}
$$

We put $V^{0}=g^{2} D_{0}$ to obtain

$$
V_{e l-p h}(\omega)=\frac{g^{2}}{[1-U \chi(\omega) / 2]^{2}} D(\omega),
$$

where $D(\omega)$ and $\chi(\omega)$ are given, respectively, by Eqs. (23) and (20). We will use the renormalized phonon propagator of Eq. (23) in the following section to understand the phonon renormalization and emergence of the soft phonon mode.

\section{SPECTRAL FUNCTIONS AND SUM RULE}

The renormalized phonon Green's function $d(\omega)$ was obtained by the method (b) of Eq. (8) of the previous section by calculating $\left\langle\left\langle n_{f}, a^{\dagger}\right\rangle_{\omega}\right.$ and $\left\langle\left\langle a, a^{\dagger}\right\rangle\right\rangle_{\omega}$ directly with the NRG technique. Some technical details of the NRG scheme can be found in Ref. [12, 13]. Briefly, the conduction electron band of the AH model, which was taken from -1 to 1 , is logarithmically discretized and mapped onto a semi-infinite fictitious chain coupled to the impurity site at one end such that the hopping amplitude along the chain falls off expontially $\left(\sim \Lambda^{-n / 2}\right)$. The many body eigen-states and energies are calculated iteratively starting from the impurity site and adding an extra site along the chain at each step. After a few steps, the number of states has to be truncated to the lowest $N_{s t}$ ones. In the present work, we used the discretization parameter $\Lambda=2.0$, kept $N_{s t}=600$ states in each iteration, and 100 fictitious sites along the chain. The lowest 40 phonon states are kept for the AH model with the bare phonon frequency $\omega_{0}=0.05$. The energy unit is the half the conduction bandwidth.

We now compare the three ways of calculating the $d(\omega)$ by the methods (a), (b), and (c), given, respectively, by Eqs. (3), (8), and (10) explained in the previous section. In

Fig. 2, we show $-\frac{1}{\pi} \operatorname{Im} d(\omega)$ vs. $\omega$ calculated by the above three methods for $U=0.1$ and $\Delta=\omega_{0} / \pi \approx 0.0159$, for the electron-phonon coupling constants $g=0.01,0.03,0.05$, 0.07, and 0.09. The NRG calculations yield the discrete frequencies; the resulting spectral functions are discrete with the $\delta$-peaks getting closer as $\omega \rightarrow 0$. The $\delta$-peaks are broadened 

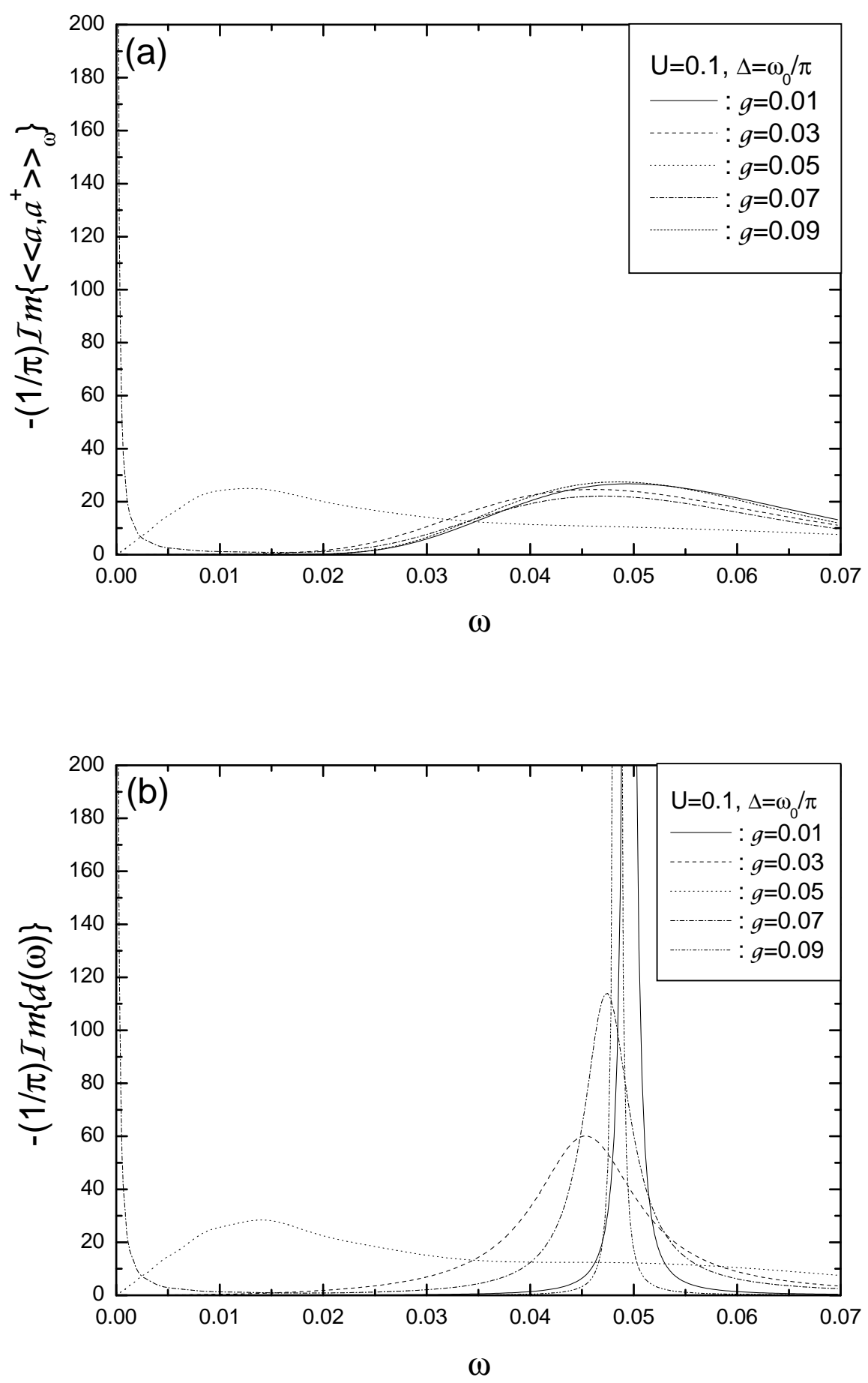


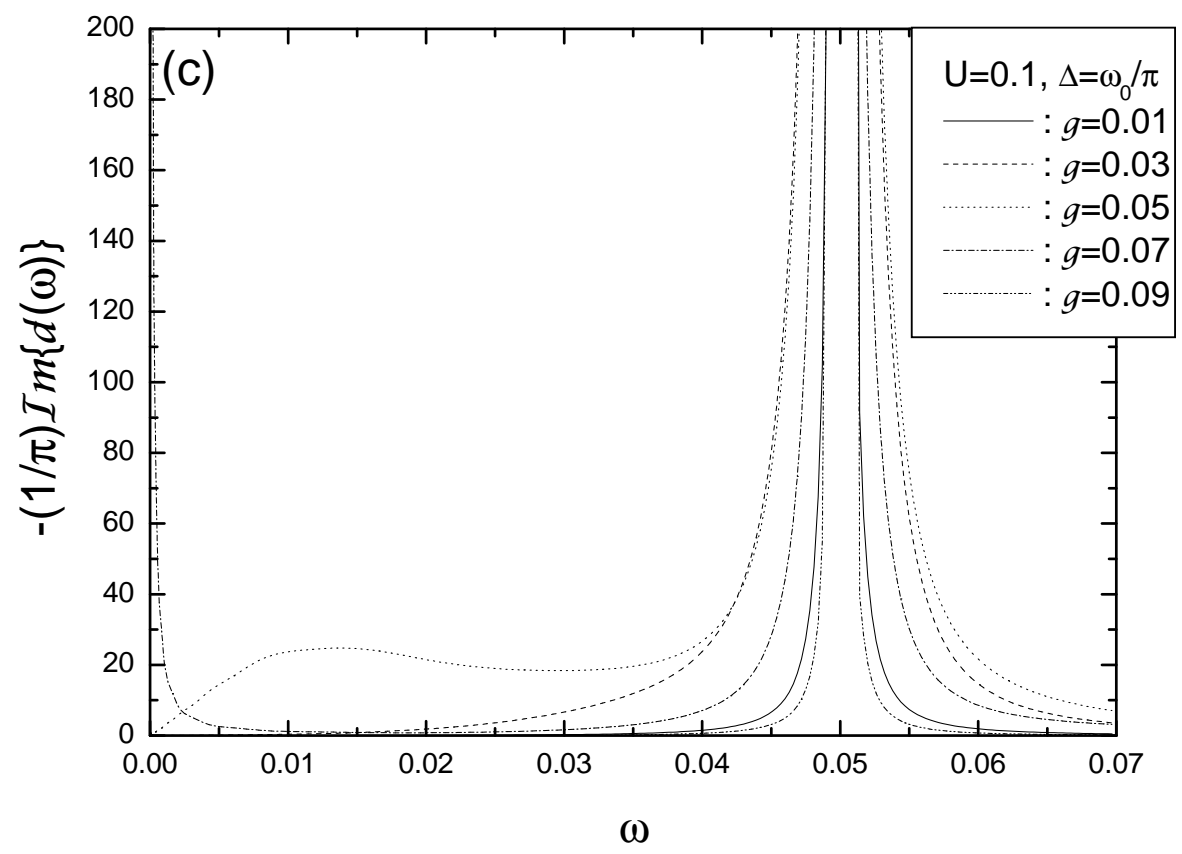

FIG. 2: The phonon spectral function $-\frac{1}{\pi} \operatorname{Im} d(\omega)$ vs. $\omega$ calculated by the three methods for $U=0.1$ and $\Delta=\omega_{0} / \pi \approx 0.0159$. Figure (a), (b), and (c) are obtained by the method (a) the direct NRG calculation of $\left\langle\left\langle a, a^{\dagger}\right\rangle_{\omega}\right.$, (b) the present calculation of Eq. (8), and (c) Hewson and Meyer of Eq. (10), respectively.

via

$$
\delta\left(\omega-\omega_{n}\right) \rightarrow \frac{e^{-b^{2} / 4}}{b \omega_{n} \sqrt{\pi}} \exp \left[-\frac{\left(\ln \omega-\ln \omega_{n}\right)^{2}}{b^{2}}\right] .
$$

This broadening function is a Gaussian on a logarithmic scale with the width $b . b=0.4$ was used in the present study. Figures (a), (b), and (c) are the results obtained by the methods (a), (b), and (c), respectively. For the low frequency, they show the same behavior, but for $\omega \approx \omega_{0}$, they show deviations from each other. In the method (a) the feature around the bare frequency $\omega_{0}$ is almost washed out, while in the method (c) the peak is always tied to the bare frequency because of the singularity of the $\left[d_{0}(\omega)\right]^{2}$. In the method (b), the phonon frequency is successively renormalized as one may expect from the perturbation theory. As the electron-phonon coupling constant $g$ is increased, the phonon mode softens until the soft mode begins to emerge around $g \approx g_{c o}$, which will be discussed below. As $g$ is further increased above $g_{c o}$, the mode splits into two components, one of which hardens back to the 


\begin{tabular}{|c|c|c|}
\hline$g$ & Direct NRG & Improved method \\
\hline 0.01 & 1.000046 & 0.999922 \\
0.03 & 0.999212 & 0.999890 \\
0.05 & 0.993977 & 0.999863 \\
0.07 & 0.911200 & 0.991001 \\
0.09 & 0.992165 & 0.999840 \\
\hline
\end{tabular}

TABLE I: The boson sum rule of Eq. (13). The second and third columns show, respectively, the integral $-\frac{1}{\pi} \int_{-\infty}^{\infty} d \omega \operatorname{Im} d(\omega)$ computed by the direct NRG calculation of $\left\langle\left\langle a, a^{\dagger}\right\rangle_{\omega}\right.$ and the improved method of Eq. (8).

bare frequency and the other develops into the soft mode as can be seen for $g=0.07$ and 0.09 of the figure (b). For $g=0.09$, the soft mode can hardly be seen because the width of the soft mode is very narrow in this energy scale.

In the table 1 , we compare the integrals $-\frac{1}{\pi} \int_{-\infty}^{\infty} d \omega \operatorname{Im} d(\omega)$ from the method (a) and (b) to check the sum rule of Eq. (13). The method (b) satisfies the sum rule better than (a), and the method (c) doesn't even converge because of the strong singularity of the $\left[d_{0}(\omega)\right]^{2}$ in Eq. (10). Because of the singularity, it will be extremely difficult to obtain the phonon renormalization around $\omega \approx \omega_{0}$ from the method (c). The Eq. (8) of method (b) is written in the form of Dyson equation where $(1 / g)\left\langle\left\langle n_{f}, a^{\dagger}\right\rangle\right\rangle_{\omega} /\left\langle\left\langle a, a^{\dagger}\right\rangle\right\rangle_{\omega}$ is the proper polarization, which gives the phonon renormalization as $g$ is increased. As $g$ is further increased, the soft mode of $\omega=0$ emerges, which may be understood in terms of the Green's function perturbation scheme discussed below. The present method (b) of Eq. (8) calculated the phonon renormalization explicitly and satisfies the sum rule better than the other methods and, therefore, give more reliable and satisfactory description of the phonon renormalization of the AH model.

The renormalized phonon frequency can be found from $D^{-1}(\omega)=0$. To investigate the emergence of the soft phonon mode, we substitute the expression Eq. (22) of $\chi(\omega)$ for $\omega \ll \Delta$ into Eq. (23) to obtain

$$
\omega^{2}\left[1-\frac{4 \pi g^{2} \omega_{0}(10 \pi \Delta-U)}{3 \Delta(2 \pi \Delta+U)^{3}}\right]-\omega_{0}\left(\omega_{0}-\frac{4 g^{2}}{2 \pi \Delta+U}\right)=0 .
$$




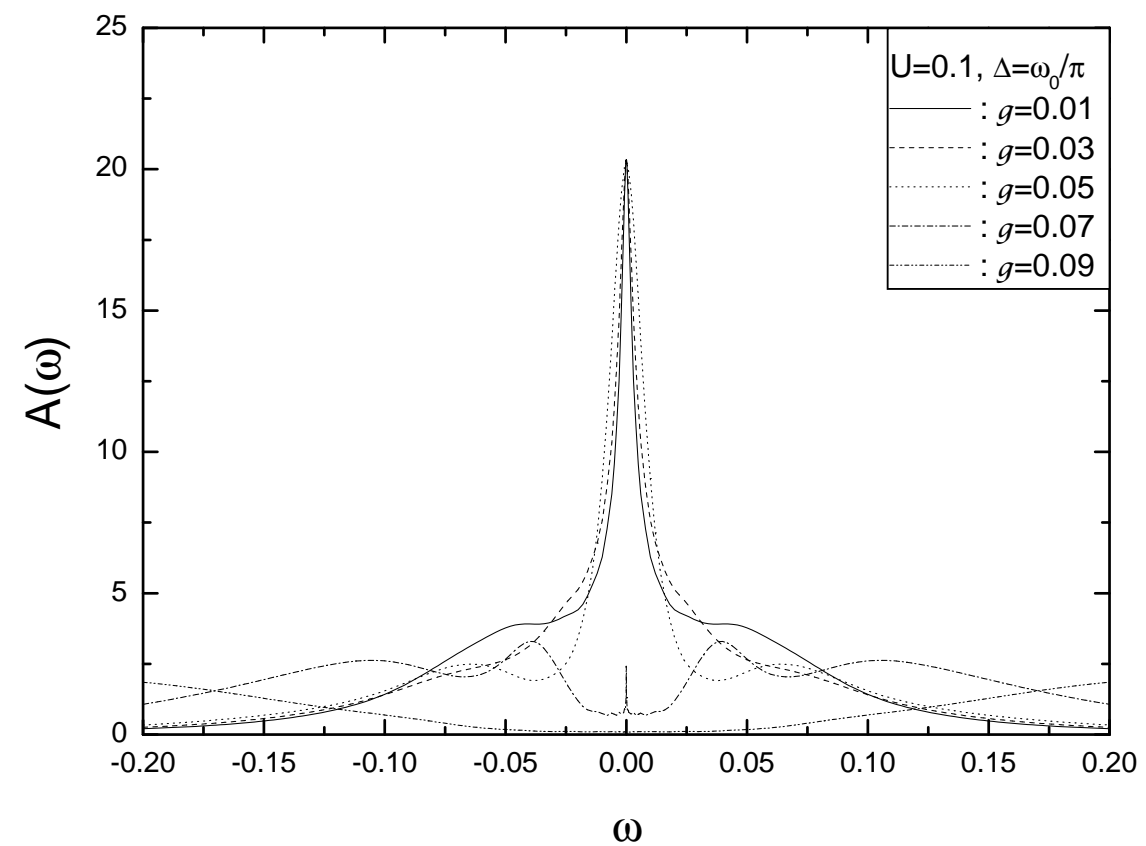

FIG. 3: The electron spectral function $-\frac{1}{\pi} \operatorname{Im} G(\omega)$ vs. $\omega$ for $U=0.1$ and $\Delta=\omega_{0} / \pi$ corresponding to the figure 2 .

The cross-over value $g_{c o}$ is then given by

$$
g_{c o}=\frac{1}{2} \sqrt{\omega_{0}(2 \pi \Delta+U)}
$$

which gives $g_{c o}=0.05$ for $U=0.1$ and $\Delta=\omega_{0} / \pi$. We therefore expect the soft mode emerges for $g \gtrsim g_{c o}$ which agrees fairly well with the NRG calculations of Fig. 2(b).

In Fig. 3, we show the electron spectral function $A(\omega)=-\frac{1}{\pi} \operatorname{Im} G(\omega)$ vs. $\omega$ for the same parameters as the Fig. 2. For small $g$ such that $\bar{U}>\pi \Delta$, where $\bar{U}=U-2 E_{p}$ is the effective onsite repulsion and $E_{p}=g^{2} / \omega_{0}$ is the polaron binding energy, the system is in the Kondo regime. The peak at $\omega=0$ is then the Kondo resonance peak whose width is given by

$$
2 T_{K} \simeq(\bar{U} \Delta)^{\frac{1}{2}} \exp \left(-\frac{\bar{U}}{\pi \Delta}\right)
$$

and the height is

$$
A(\omega=0)=\frac{1}{\pi \Delta}
$$

in agreement with the figure 3 . The shoulders at $\omega \approx \pm \bar{U} / 2 \approx \pm 0.05$ correspond to the charge fluctuations at the $f$ electron impurity site. As $g$ is increased, $\bar{U}$ is decreased, and the 
width of the Kondo peak increases as can be seen from the figure. The effective $\bar{U}$ becomes negative for $g>g_{-}=\sqrt{U \omega_{0} / 2} \cdot g_{-}=0.05$ for $U=0.1$ and $\omega_{0}=0.05$. The electron spectral function in this case may be understood in terms of the small polaron picture as discussed by Hewson and Meyer [11]. The electron Green's function for small hybridization limit of $\Delta \rightarrow 0$ is given by

$$
\begin{array}{r}
G_{\sigma}(\omega)=e^{-\lambda} \sum_{n=0}^{\infty} \frac{\lambda^{n}}{n !}\left[\begin{array}{r}
\left\langle\left(1-n_{f, \sigma}\right)\left(1-n_{f,-\sigma}\right)\right\rangle \\
\omega-\bar{\epsilon}_{f}-n \omega_{0}
\end{array}+\frac{\left\langle\left(1-n_{f, \sigma}\right) n_{f,-\sigma}\right\rangle}{\omega-\bar{\epsilon}_{f}-\bar{U}-n \omega_{0}}\right. \\
\left.+\frac{\left\langle n_{f, \sigma}\left(1-n_{f,-\sigma}\right)\right\rangle}{\omega-\bar{\epsilon}_{f}+n \omega_{0}}+\frac{\left\langle n_{f, \sigma} n_{f,-\sigma}\right\rangle}{\omega-\bar{\epsilon}_{f}-\bar{U}+n \omega_{0}}\right],
\end{array}
$$

where $\lambda=g^{2} / \omega_{0}^{2},\left\langle n_{f, \sigma}\right\rangle=\left\langle f_{\sigma}^{\dagger} f_{\sigma}\right\rangle$, and $\bar{\epsilon}_{f}=\epsilon_{f}+E_{p}$. Note that $\bar{\epsilon}_{f}=\epsilon_{f}+E_{p}$ instead of $\bar{\epsilon}_{f}=\epsilon_{f}-E_{p}$ because the coupling between the $f$ electrons and the phonons is taken to be proportional to $\left(\sum_{\sigma} f_{\sigma}^{\dagger} f_{\sigma}-1\right)$ instead of $\left(\sum_{\sigma} f_{\sigma}^{\dagger} f_{\sigma}\right)$. The set of delta functions at $\omega=\bar{\epsilon}_{f}+n \omega_{0}, \bar{\epsilon}_{f}-n \omega_{0}, \bar{\epsilon}_{f}+\bar{U}-n \omega_{0}$ and $\bar{\epsilon}_{f}+\bar{U}-n \omega_{0}$ for integer $n$, determined by the denominators of Eq. (35), will be broadened for non-zero $\Delta$. The most probable phonon occupation number is $\langle n\rangle=E_{p} / \omega_{0}=\lambda$. For integer $n$ 's, the peaks are expected at

$$
\begin{aligned}
& \omega=\bar{\epsilon}_{f} \pm n \omega_{0}=\epsilon_{f}+E_{p}, \epsilon_{f}+E_{p} \pm \omega_{0}, \cdots, \\
& \omega=\bar{\epsilon}_{f}+\bar{U} \pm n \omega_{0}=\epsilon_{f}+U-E_{p}, \epsilon_{f}+U-E_{p} \pm \omega_{0}, \cdots,
\end{aligned}
$$

which roughly tracks the four high energy peaks of the spectral function $A(\omega)$ as $g$ is increased. For $g=0.07$ for instance, $E_{p}=0.098$, and we expect the peaks at $\omega= \pm 0.048, \pm 0.098$, and so forth, which roughly agree with the detailed NRG results shown in the figure 3. The central peak for the negative $\bar{U}$ may be understood in terms of the Kondo-like resonance of the negative $U$ model, which arises because of the charge fluctuations, instead of the spin fluctuations in the Kondo regime, between the zero and double occupation states at the impurity site. When $g$ is further increased above $g_{c o}$, the soft phonon mode emerges. The effective $|\bar{U}|$, which equals to $2 g^{2} / \omega_{0}-U$ for the Einstein phonon may be generalized to

$$
|\bar{U}|=2 g^{2}\left\langle\frac{1}{\omega}\right\rangle-U, \text { where }\left\langle\frac{1}{\omega}\right\rangle=-\int_{-\infty}^{\infty} d \omega \frac{1}{\pi} \operatorname{Im} d(\omega) \frac{1}{\omega} .
$$

When the soft mode emerges, the $\bar{U}$ can be very large and the width of the electron central peak given by Eq. (33) becomes very narrow. The central peak can hardly be seen for $g=0.07$ and 0.09 of the Fig. 3 in this frequency scale. 

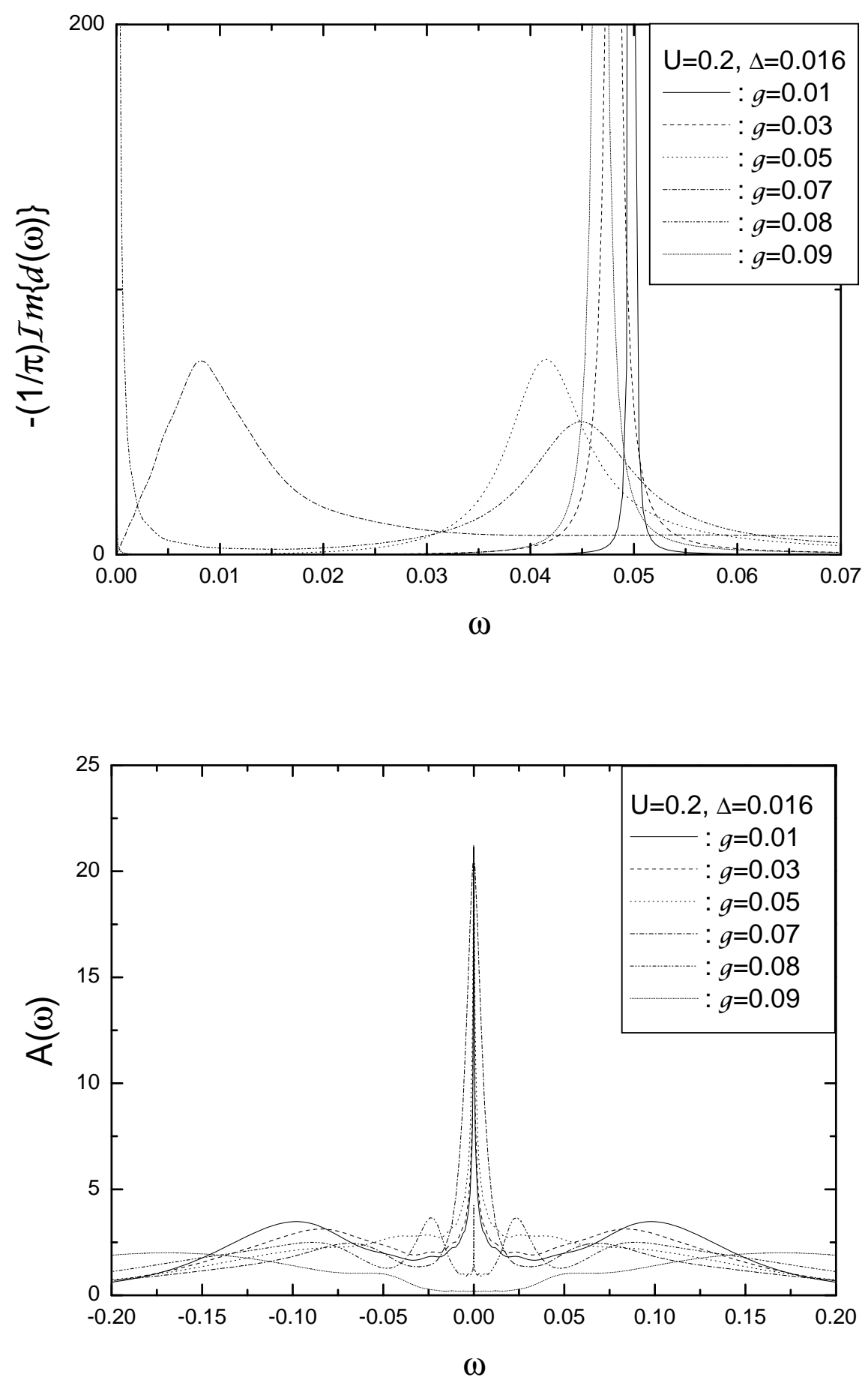

FIG. 4: The phonon and electron spectral functions vs. $\omega$ for $U=0.2$ and $\Delta=0.016$. 

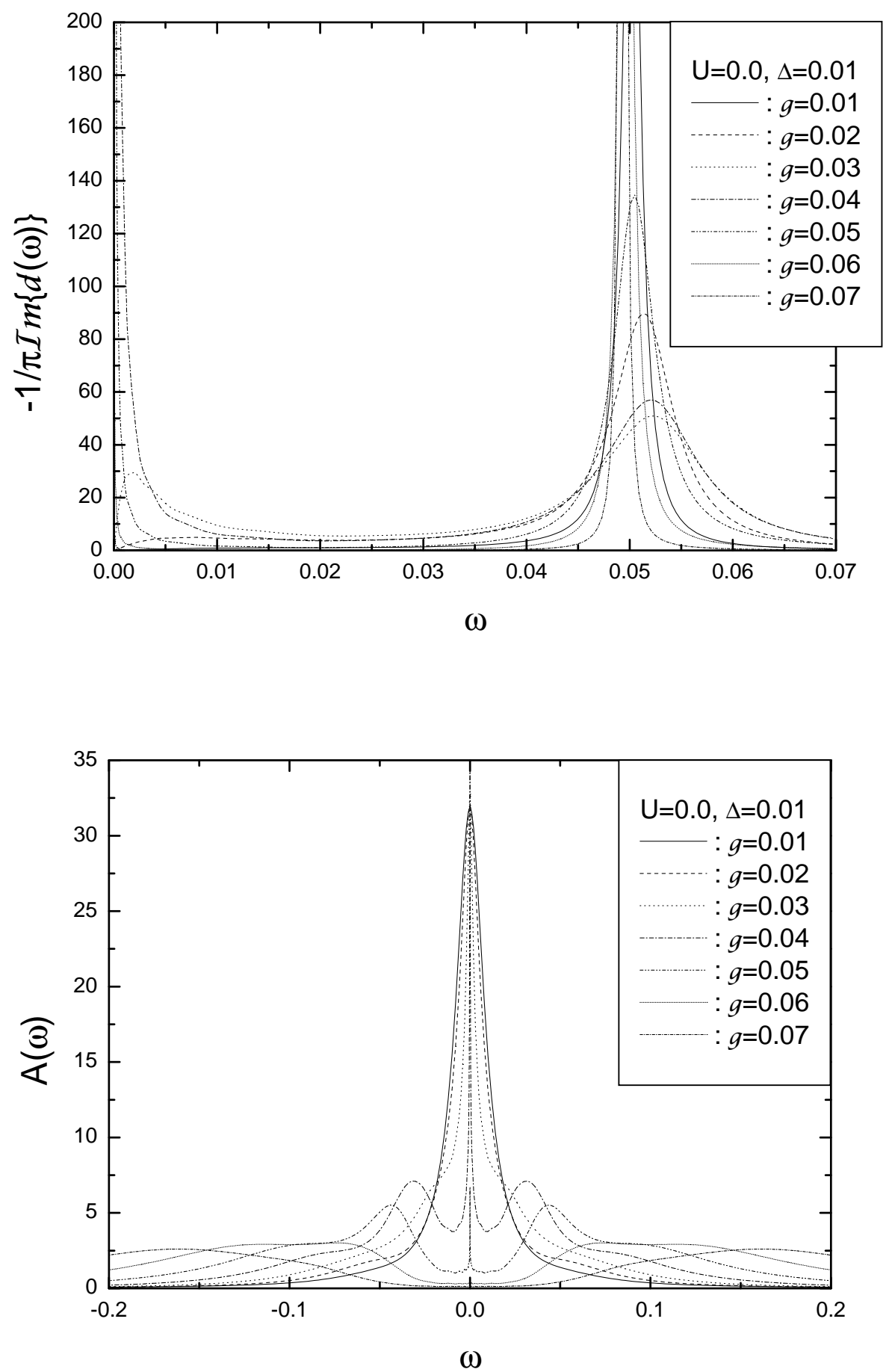

FIG. 5: The phonon and electron spectral functions vs. $\omega$ for $U=0$ and $\Delta=0.01$. 
We now present the results calculated with the method (b) for other parameter values. Fig. 4 is for $U=0.2$ and $\Delta=0.016 \approx \omega_{0} / \pi$, and Fig. 5 is for $U=0$ and $\Delta=0.01$. They exhibit the similar behaviors as for the $U=0.1$ and $\Delta=\omega_{0} / \pi$ of figures 2 and 3 . The emergence of the soft phonon modes and suppression of the central peak are correlated for both cases and agree with the estimate of the perturbation theory of Eq. (32) as discussed above. $g_{c o}=0.0613$ for $U=0.2$ and $\Delta=0.016$ and $g_{c o}=0.0280$ for $U=0$ and $\Delta=0.01$ which are consistent with the NRG results of figures 4 and 5, respectively. The four high energy peaks for negative $\bar{U}$ are also consistent with the small polaron estimate of Eq. (36). For $U=0.2$, shown in Fig. $4, \epsilon_{f}=-0.1$, and $E_{p}=0.128$ for $g=0.08$. We therefore expect to see the peaks in the electron spectral function at $\omega \approx \pm\left(E_{p}+\epsilon_{f}\right), \pm\left(E_{p}+\epsilon_{f}+\omega_{0}\right), \cdots \approx$ $\pm 0.028, \pm 0.078$, and so forth, which agree with Fig. 4. For $U=0$, shown in Fig. 5, $\epsilon_{f}=0$, and $E_{p}=0.032$ and 0.05 for $g=0.04$ and 0.05 , respectively. The peaks are expected at $\omega \approx \pm 0.032$ and \pm 0.082 for $g=0.04$, and at $\omega \approx \pm 0.05$ and \pm 0.1 for $g=0.05$, which are again consistent with the electron spectral function $A(\omega)$ shown in Fig. 5. The heights of the central peak of the electron spectral function $A(\omega)$ are given by $1 /(\pi \Delta)$ of Eq. (34) for both cases.

For $U=0$, the phonon renormalization seems somewhat different from the $U \neq 0$ cases of Figs. 2 and 4 in that the main peak around $\omega_{0}$ is slightly hardened as $g$ is increased. This apparent difference can be straightforwardly understood from the Green's function perturbation results presented in the previous section. If one solves for the renormalized phonon frequency from $D^{-1}(\omega)=0$ using Eqs. (23) and (20), one can see that the renormalized frequency gets softened or hardened depending on $\Delta$ and $U$. The value of $U_{s}$ above (below) which the renormalized phonon frequency, as $g$ in increased from 0 , is softened (hardened) is simply given by $\operatorname{Re}\left[\chi\left(\omega_{0}\right) /\left(1-(U / 2) \chi\left(\omega_{0}\right)\right)\right]=0$ from Eq. (23), which yields

$$
U_{s}(\Delta)=2 \frac{\operatorname{Re} \chi\left(\omega_{0}\right)}{\left|\chi\left(\omega_{0}\right)\right|^{2}}=\frac{2 \pi \omega_{0}^{2}}{\Delta} \frac{\ln \left(1+\frac{\omega_{0}^{2}}{\Delta^{2}}\right)-4\left(\frac{\Delta}{\omega_{0}}\right) \tan ^{-1}\left(\frac{\omega_{0}}{\Delta}\right)}{\ln ^{2}\left(1+\frac{\omega_{0}^{2}}{\Delta^{2}}\right)+4 \tan ^{-2}\left(\frac{\omega_{0}}{\Delta}\right)} .
$$

For $\omega_{0}=0.05$, one finds that $U_{s}=0.0636$ for $\Delta=\omega_{0} / \pi$, and 0.1868 for $\Delta=0.01$. We therefore expect that the phonon renormalization will get softened for $U=0.1$ and $\Delta=\omega_{0} / \pi$, and $U=0.2$ and $\Delta=0.016 \approx \omega_{0} / \pi$, corresponding, respectively, to Fig. 2(b) and Fig. 4, but hardened for $U=0$ and $\Delta=0.01$ corresponding to Fig. 5 , in agreement with the NRG results presented in those figures.

Note, however, that the phonon spectral function of $U=0$ has a long tail towards $\omega=0$ 
compared with the $U \neq 0$ cases and, therefore, the average phonon frequency gets softened as $g$ is increased. When $g \gtrsim g_{c o}$, the soft mode emerges in addition to the peak around $\omega_{0}$, and the average phonon frequency hardens back to the bare frequency of $\omega_{0}$ as $g$ is further increased above $g_{c o}$ like the $U \neq 0$ cases. Also noticeable is that the hardening or softening of the renormalized phonon frequency for $U=0$ does depend on $\Delta$. From Eq. (38), we expect that the renormalized frequency will get hardened (softened) for $\Delta<(>$ ) $0.410 \omega_{0}=0.0205$. The NRG results presented in Fig. 5 for $U=0$ and $\Delta=0.01$ are consistent with the estimate.

\section{SUMMARY AND CONCLUDING REMARKS}

In the present paper, we have presented Wilson's numerical renormalization group calculations of the symmetric Anderson-Holstein model. The phonon and electron spectral functions were computed as the electron-electron interaction $U$ and electron-phonon coupling $g$ were varied. The electron spectral functions are in good agreement with the Hewson and Meyer results. On the other hand, an improved method was presented for calculating the phonon spectral functions and the results were compared with Hewson and Meyer's. Unlike the method (c) of Eq. (10) where the phonon frequency is always tied to the bare frequency, the present calculations show that as $g$ is increased, the phonon mode is renormalized until the soft mode emerges around $g \approx g_{c o}$, and as $g$ is further increased, the phonon mode splits into two components, one of which approaches back to the bare frequency and the other develops into the soft mode. We argue that the above behavior of the phonon renormalization is physically more sensible and is a correct picture in that (a) in the present method the phonon renormalization is explicitly computed, and (b) the present method satisfies the boson sum rule better than the previous methods as listed in Table 1. These NRG calculations were also compared with the standard Green's function results for the weak coupling regime to understand the phonon renormalization and soft mode.

Now that the calculation of the dynamical properties of the impurity Anderson-Holstein model is successfully carried out, we plan to extend these calculations to the lattice model of the Hubbard-Holstein model within the framework of the dynamical mean-field theory. These calculations will be relevant to high- $T_{c}$ superconductors where recent photoemission

experiments give evidence of strong electron-phonon coupling [14 and to the manganites [15]. 
Extension to the finite temperatures for the impurity model and for the lattice model should also be fruitful.

We would like to thank Hyun Cheol Lee, Tae Suk Kim, Ralf Bulla, Dietrich Meyer, and Alex Hewson for helpful comments and discussions. This work was supported by the Korea Science \& Engineering Foundation (KOSEF) through grant No. R01-1999-000-00031-0 and Center for Nanotubes and Nanoscale Composites (CNNC), and by the Ministry of Education through BK21 SNU-SKKU Physics program.

[1] A. Deppeler and A. J. Millis, Phys. Rev. 65, 100301 (2002).

[2] W. Metzner and D. Vollhardt, Phys. Rev. Lett. 62, 324 (1989); ibid, 1066 (1989).

[3] A. Georges, G. Kotliar, W. Krauth, and M. J. Rozenberg, Rev. Mod. Phys. 68, 13 (1996).

[4] H. R. Krishna-murthy, J. W. Wilkins, and K. G. Wilson, Phys. Rev. B 21, 1003 (1980).

[5] H. R. Krishna-murthy, J. W. Wilkins, and K. G. Wilson, Phys. Rev. B 21, 1044 (1980).

[6] K. G. Wilson, Rev. Mod. Phys. 47, 773 (1975).

[7] R. Bulla, Adv. Solid State Phys. 40, 169 (2000).

[8] R. Bulla, Phys. Rev. Lett. 83, 136 (1999).

[9] R. Bulla, T. A. Costi, and D. Vollhardt, Phys. Rev. B 64, 045103 (2001).

[10] G. D. Mahan, Many-Particle Physics, 3rd ed. (Plenum, New York, 2000).

[11] A. C. Hewson and D. Meyer, J. Phys.: Condens. Matter 14, 427 (2002).

[12] D. Meyer, A. C. Hewson, and R. Bulla, Phys. Rev. Lett. 89, 196401 (2002).

[13] R. Bulla, A. C. Hewson, and Th. Pruschke, J. Phys.: Condens. Matter 10, 8365 (1998).

[14] A. Lanzara, P. V. Bogdanov, X. J. Zhou, S. A. Keller, D. L. Feng, E. D. Lu, T. Yoshida, H. Eisaki, A. Fujimori, K. Kishio, J.-I. Shimoyama, T. Nodaand S. Uchida, Z. Hussain, and Z.-X. Shen, Nature 412, 510 (2001).

[15] A. P. Ramirez, J. Phys.: Condens. Matter 9, 8171 (1997).

[16] A. J. Millis, P. B. Littlewood, and B. I. Shraiman, Phys. Rev. Lett. 74, 5144 (1995). 